\title{
S100A12 and RAGE Expression in Human Bladder Transitional Cell Carcinoma: a Role for the Ligand/RAGE Axis in Tumor Progression?
}

\author{
Hossein Khorramdelazad ${ }^{1,2}$, Vahid Bagheri ${ }^{1}$, Gholamhossein Hassanshahi ${ }^{1,2}$ \\ Hormoz Karami ${ }^{3}$, Mozhgan Moogooei ${ }^{1,2}$, Masoud Zeinali ${ }^{4}$, Mehdi Abedinzadeh ${ }^{3 *}$
}

\begin{abstract}
Background: Transitional cell carcinoma (TCC) and prostate cancer are the most frequent cancers in the male genitourinary tract. Measurement of biological biomarkers may facilitate clinical monitoring and aid early diagnosis of TCC. The aim of the present investigation was to detect the mRNA levels of S100A12 and RAGE (receptor for advanced glycation end products) in patients suffering from bladder TCC. Materials and Methods: To explore the involvement of S100A12 and RAGE genes, total RNA was harvested from cancer tissues and samples obtained from normal non-tumorized urothelium of the same patients. Quantitative PCR (qPCR) was subsequently employed to determine the mRNA levels of S100A12 and RAGE. Results: The results showed that mRNA expression of S100A12 and RAGE was significantly up-regulated in the cancer tissue. Conclusions: According to the results presented in the current study, mRNA expression of S100A12 and RAGE might be as a useful biomarker for TCC. Therefore, this ligand-receptor axis possibly plays important roles in the development of TCC and may serve either as an early diagnostic marker or as a key factor in monitoring of response to treatment. More research is required concerning inhibition of the S100A12-RAGE axis in different cancer models.
\end{abstract}

Keywords: TCC - bladder cancer - S100A12 - RAGE

Asian Pac J Cancer Prev, 16 (7), 2725-2729

\section{Introduction}

Bladder cancer causes approximately 150,000 fatalities annually. Among environmental risk factors, tobacco smoking is of primary importance (Knowles and Hurst, 2015). TCC accounts for a significant proportion of bladder cancer (about 90\%) (Gui et al., 2011). In south Asia, Pakistan has the highest rate of bladder cancer and $72 \%$ of cases in Sri Lanka are TCC form (Ranasinghe et al., 2012). Bladder cancer comprises $7.04 \%$ of cancers in Iran, according to reports of the Iranian Center for Disease Control and Prevention.

One of the main risk factors for bladder cancer in Iran has been found to be opium use (Shakhssalim et al., 2010). The prevalence of bladder cancer has also been reported in Shiraz, south of Iran, and the most common type of the disease was TCC $(95.7 \%$ of all cases) (Salehi et al., 2011). It has been suggested that several inflammatory cytokines may play a role in migration and invasion of TCC (Lee et al., 2012). S100 family members are EF-hand calcium-binding proteins which perform a variety of different cellular functions including cell invasion, cell proliferation, apoptosis, autoimmunity, and inflammation. Several S100 proteins also act as ligands for RAGE and Toll-like receptors (TLRs). Subsequently, extracellular S100 proteins interact with RAGE resulting in activation of signaling pathways such as MAPK, PI-3K-AKT, and NF- $x$ B. Due to their roles in multiple cellular processes, $\mathrm{S} 100$ proteins are of vital importance in developing cancers (Pietzsch, 2011; Chen et al., 2014). RAGE, which is a transmembrane receptor, is a member of immunoglobulin superfamily. It is also a crucial factor in the development of various diseases, including cancer, cardiovascular diseases, and diabetes (Xie et al., 2013; Sessa et al., 2014). The S100A12 (Calgranulin C), which is mainly expressed by neutrophils and monocytes, is associated with innate immune responses. However, it can be expressed in particular cell types, including epithelial cells, and muscle cells during inflammation and carcinogenesis. After binding of the S100A12 to the RAGE, S100A12-RAGE axis triggers intracellular signaling pathways that induce production of proinflammatory cytokines and adhesion molecules. As a result, studies have shown that the S100A12 can be

${ }^{1}$ Molecular Medicine Research Center, ${ }^{2}$ Department of Immunology, ${ }^{4}$ Department of Social Medicine, Faculty of Medicine, Rafsanjan University of Medical Sciences, Rafsanjan, ${ }^{3}$ Department of Urology, Shahid Rahnemoon Hospital, Shahid Sadoughi University of Medical Sciences and Health Services, Yazd,Iran*For correspondence: abedinoro@yahoo.com 
useful as diagnostic biomarker for inflammatory diseases (Meijer et al., 2012; Funk et al., 2014).

The present study was aimed to explore whether S100A12-RAGE axis may play an important part in the pathogenesis of TCC. Therefore, the study investigates S100A12-RAGE gene expression at the transcriptional level in bladder TCC tissues in comparison with normal tissues.

\section{Materials and Methods}

\section{Subjects}

In the present cross-sectional study, we have recruited 17 patients suffering from TCC who referred to Shahid Bahonar Hospital of Kerman University of Medical Sciences during 2012-13 (Table2). The diagnosis of TCC was confirmed by an expert urologic surgeon based on sonography and pathological evidences. Patients who exhibited evidence for any type of inflammatory disorder, infection, and chronic disease as well as other types of cancer were excluded from the study. Both of tumoral and normal bladder tissues were collected from patients once they had been diagnosed and during the processes of clinical diagnosis. After tissue collection, all samples were promptly placed in liquid nitrogen and then stored at $-80^{\circ} \mathrm{C}$ until RNA isolation. The human ethical approval for this study was granted by the ethical committee of Rafsanjan University of Medical Sciences and written informed consent was obtained from all of patients, before initiation of study program.

\section{$R N A$ extraction, reverse transcription, and $q P C R$}

Total cellular RNA was extracted from tissues using Total RNA Purification Kit (Jena Bioscience, Germany) according to the manufacturer's instructions. The purity and fidelity of RNA were examined following electrophoresis on ethidium bromide pretreated agarose gel as well as absorption ratio at $260 / 280 \mathrm{~nm}$ via a spectrophotometric method. The resultant RNA was then converted to cDNA using AccuPower RocketScript RT PreMix (Bioneer, South Korea) with both oligo (dT) and random hexamer primers. The following protocol was applied for reverse transcription and the generation of cDNA: $70^{\circ} \mathrm{C}$ for $10 \mathrm{~min}$ (without reverse transcription enzyme), $-20^{\circ} \mathrm{C}$ for $1 \mathrm{~min}$ (cooling step), addition of reverse transcription enzyme, $42^{\circ} \mathrm{C}$ for $60 \mathrm{~min}$, and $95^{\circ} \mathrm{C}$ up to $10 \mathrm{~min}$ to terminate the further activation of the reverse transcription enzyme. Real-time qPCR was performed using AccuPower GreenStar qPCR Master Mix (Bioneer, South Korea), combined with $200 \mathrm{ng}$ of resultant cDNA with the appropriate primers in a Bio-Rad CFX96 system (Bio-Rad Company, USA) using the following program: 1 cycle of $95^{\circ} \mathrm{C}$ for $15 \mathrm{~min}$ and 40 cycles of $95^{\circ} \mathrm{C}$ for $30 \mathrm{~s}, 60^{\circ} \mathrm{C}$ for $30 \mathrm{~s}$, and finally $72^{\circ} \mathrm{C}$ for $30 \mathrm{~s}$. Realtime PCR was carried out in triplicate and the $\beta$-actin was applied as a housekeeping gene for normalization of the amplified signal of the target genes. The oligonucleotide primers for qPCR were synthesized by Bioneer, Sought Korea. Table 1 indicates the sequences of the primers used in this study. The relative expression levels of the S100A12 and RAGE were determined by using the $2^{-\Delta \Delta \mathrm{Ct}}$ method.
The dissociation stages, melting curves and quantitative analyses of the data were performed using CFX manager software version 1.1.308.111 (Bio-Rad, USA). PCR products were also analyzed by electrophoresis and visualized on a $1 \%$ agarose gel containing $0.5 \mathrm{mg} / \mathrm{ml}$ ethidium bromide.

\section{Statistical analysis}

Statistical analysis of the differences was performed by employing the statistical package for the social sciences (SPSS, Inc., Chicago, IL, USA) version 18. Data were then analyzed using T test and Mann-Whitney test. The $\mathrm{P}$-values less than 0.05 were considered significant.

\section{Results}

We enrolled 17 men with bladder TCC from 51-61 years of age. Table 2 summarizes demographic and other features of patients with TCC, including exposure to chemicals, smoking, and marital status. Environmental risk factors for bladder cancer in 17 patients were smoking $(88.2 \%)$, and exposure to chemicals $(64.7 \%$ with more than 10 years).

In order to confirm RNA integrity and purity, electrophoresis and spectrophotometry methods were used. Absorption ratio at 260/280 nm for all RNA samples was between 1.8 and 2. Figure 1 shows total RNA samples loaded on $1 \%$ agarose gel. After electrophoresis, $28 \mathrm{~S}$ and $18 \mathrm{~S}$ ribosomal RNA bands were observed.

In this study, real-time quantitative PCR (qPCR) was selected as a sensitive method for quantifying the expression of S100A12 and RAGE genes in TCC tissues. An increase in mRNA levels of S100A12 and

\section{Table 1. List of Primers Used for qPCR}

\begin{tabular}{lll}
\hline Gene & Forward primer & Reverse primer \\
\hline S100A12 & GGAGGGAATT & ATCTTGATTA \\
& GTCAATATC & GCATCCAGG \\
RAGE & GACCCTGGAA & CCCCTTACAC \\
& GGAAGCAG & TTCAGCACC \\
\multirow{2}{*}{-actin } & ACTCTTCCAG & TACAGGTCTT \\
& CCTTCCTT & TGCGGATG \\
\hline
\end{tabular}

*RAGE, Receptor of advanced glycation end products

Table 2. Demographic Characteristics and History of Risk Factors in TCC Patients. Chemical Exposure is Associated with being Exposed to either dyes or Vehicle Fumes

\begin{tabular}{llc}
\hline & & No. of patients \\
\hline Sex & Male & 17 \\
& Female & 0 \\
Age (years) & $\leq 55$ & 2 \\
& $55-58$ & 5 \\
& $59-60$ & 4 \\
Marital status & $>61$ & 6 \\
& Single & 1 \\
Smoking behavior & Married & 16 \\
& Never & 2 \\
& Former & 5 \\
Exposure to chemicals & Current & 10 \\
& $\leq 10$ years & 6 \\
& $\geq 10$ years & 11 \\
\hline
\end{tabular}




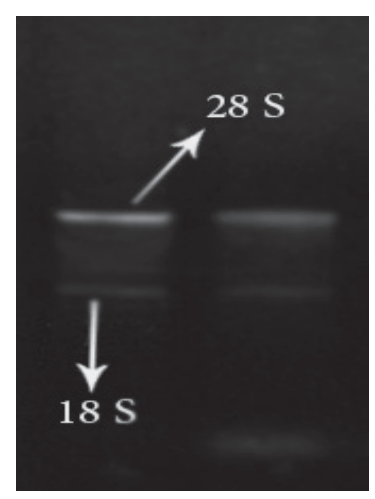

Figure 1. Total RNA Extracted from Tumor and Normal Tissue Samples

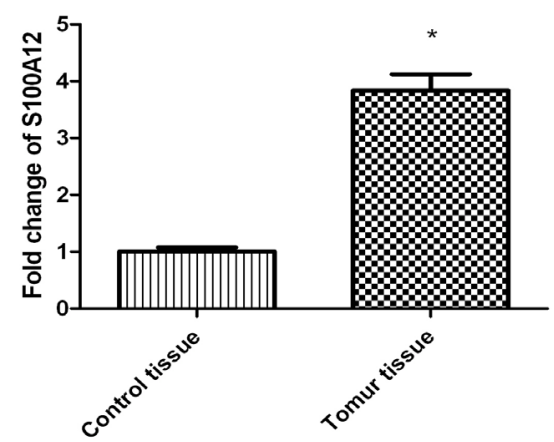

Figure 2. Expression of S100A12 at mRNA Level in TCC Tissues in Comparison to Controls. Values are expressed as mean \pm SEM for all experiments. [ ${ }^{*} \mathrm{p}<0.05$ versus relevant control tissue]

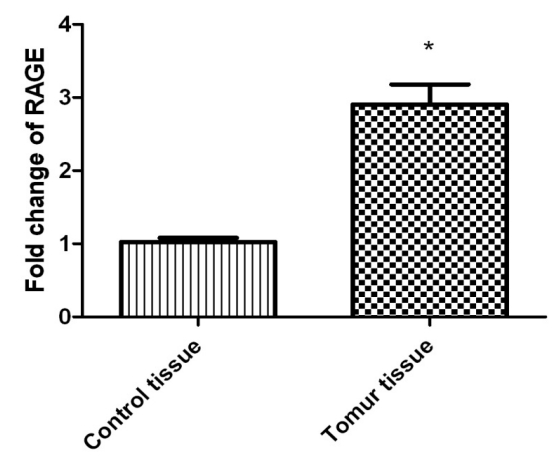

Figure 3. Expression of RAGE at mRNA Level in TCC Tissues in Comparison to Controls. Values are expressed as mean \pm SEM for all experiments. [ $* \mathrm{p}<0.05$ versus relevant control tissue]

RAGE was observed in cancerous tissue of TCC patients when compared to relative normal tissue. Our results indicate that in tumor tissues, the S100A12 expression was significantly increased by $3.83 \pm 0.28$ folds $(\mathrm{p}<0.05)$ (Figure 2) whilst RAGE expression was elevated by $2.9 \pm 0.2$ folds in tumor tissues compared to normal ones $(\mathrm{p}<0.05)$ (Figure 3).

\section{Discussion}

The S100 family proteins are described as proteins with EF-hand calcium-binding motifs which are able to regulate many intracellular and extracellular activities (Donato et al., 2013). It is now well established that up-regulation of several S100 proteins is involved in the progression of numerous cancers (Salama et al., 2008).
Recently, S100 genes and proteins such as S100A8 and S100A9 have been identified to have a major role in progression and invasion of breast cancer (McKiernan et al., 2011; Yin et al., 2013). Additionally, a study showed that there was a link between increased mRNA levels of S100A4 and metastasis in osteosarcoma (Zhang et al., 2011). High S100A14 expression has been shown to be involved in invasion and metastasis of hepatocellular carcinoma (HCC) (Zhao et al., 2013). The S100A2 has been found to be significantly diminished in cancer cells and postulated to be a tumor suppressor (Nagy et al., 2001). Lack of S100A2 and increased expression of S100A4 has been implicated in prostate tumor progression (Gupta et al., 2003). On the other hand, decreased expression of S100A2 and increased expression of S100A4 were also found in bladder TCC. The report concluded that S100A2 and S100A4 expression is a determining factor in tumor progression (Matsumoto et al., 2007). Down-regulated levels of S100A8 and S100A9 has also been reported in esophageal squamous cell carcinoma (ESCC) (Luo et al., 2004). Considerable decrease in S100A11 (S100C) mRNA in invasive bladder tumors (stages T1-T4) compared with superficial tumors (stage Ta) has been shown. Moreover, there was an association between diminished expression of S100C and poor survival in patients with bladder cancer (Memon et al., 2005).

A number of S100 proteins such as S100A4, S100A8, S100A9, S10012, and S100A13 have extracellular functions acting in a cytokine-like manner, which the mechanism of their secretion has yet to be described (Donato, 2003; Fritz, 2011). Studies conducted on the relationship between expression of S100 proteins and bladder cancer in animal and human models have demonstrated that these proteins are connected with tumor invasion and metastasis. However, only limited studies are found addressing the role of $\mathrm{S} 100$ proteins in the pathogenesis of bladder cancer. Thus, specific molecular functions of S100 proteins in bladder cancer warrant further investigation into this area (Yao et al., 2007a). A recently published paper proposed that urinary S100A12 could be useful for detecting TCC in dogs (Heilmann et al., 2014). Moreover, Yao et al. (2007) assessed the S100 genes expression in human bladder TCC by real-time PCR. Among genes reported with altered expression, S100A2, S100A3, S100A5, S100A7, S100A8, S100A9, S100A14, S100A15, S100A16 and S100P were overexpressed.

On the other hand, RAGE contributes to cancer pathogenesis and progression, as evidenced by a number of studies. An in vitro study showed that S100A7-RAGE interaction results in induction of osteosarcoma cell migration and invasion (Kataoka et al., 2012). RAGE protein and mRNA levels have been found to be elevated in hepatocellular carcinoma (Yaser et al., 2012). The new results have confirmed that expression of RAGE and its ligand, high mobility group box 1 (HMGB1), could be related to prostate cancer progression and survival (Zhao et al., 2014). Hence, there is convincing evidence of prostate cancer inhibition by silencing RAGE gene expression (Elangovan et al., 2012). In addition to gene silencing, tumor progression was suppressed by RAGE gene deletion in a mouse model, leading to the increased survival in 
pancreatic cancer (DiNorcia et al., 2012). Interestingly, a RAGE synthetic antagonist peptide, which prevents binding of ligands to RAGE, has been revealed to reduce progression and metastasis of tumors in vivo (Arumugam et al., 2012). RAGE is, therefore, a potential molecular target for cancer prevention and therapy.

Diagnosis of bladder cancer can be made by voided urine cytology and cystoscopy, however, these are not ideal detection methods due to invasion and low sensitivity (Yao et al., 2007b). Undoubtedly, appropriate molecular diagnostic markers are urgently required to improve the detection of bladder cancer and then to prolong survival of patients. The present investigation was aimed to explore the expression of S100A12/RAGE receptor-ligand pair genes in TCC patients at the mRNA level with real-time PCR. Our results showed elevated expression of the genes encoding S100A12 and RAGE proteins in tumor tissues when compared with their normal counterparts. These data suggest that S100A12 and RAGE ligandreceptor axis play significant roles in the pathogenesis and progression of TCC. There are multiple background reasons to delineate an association between the $\mathrm{S} 100$ gene family with the pathogenesis and development of TCC in humans. However, in contrast to our results, Yao et al. reported that S100A12 was not elevated in bladder cancers. The discrepancy between our results and those of Yao et al. could probably be related to both genetic and environmental parameters varying greatly from ethnic to inheritance.

Further comparative studies are needed to assess the utility of S100A12 protein as a remarkable biological marker of bladder cancer. We strongly recommend assessing the expression of other members of the S100 family, along with their receptors in these patients. Hence, the authors also propose the detection of these inflammatory mediators at both mRNA and protein level in patients suffering from bladder cancer. To our knowledge, this is the first study to address the involvement of S100A12/RAGE in the pathogenesis of bladder cancer.

To sum up, our findings raised the possibility of identifying tumor-related markers which may prove to be helpful in detecting of bladder cancer. Furthermore, these potential biomarkers may be targets for molecularbased therapeutic strategies in the prevention and/or management of bladder cancer. Future studies should explore molecular mechanisms of S100 proteins to define their roles in tumorigenesis.

\section{Acknowledgements}

The authors wish to thank all of patients for participating in this research project. This work was financially supported by a grant from Rafsanjan University of Medical Sciences.

\section{References}

Arumugam T, Ramachandran V, Gomez SB, Schmidt AM, Logsdon CD (2012). S100P-derived RAGE antagonistic peptide reduces tumor growth and metastasis. Clin Cancer Res, 18, 4356-64.
Chen H, Xu C, Jin Q, Liu Z (2014). S100 protein family in human cancer. Am J Cancer Res, 4, 89-115.

DiNorcia J, Lee MK, Moroziewicz DN, et al (2012). RAGE gene deletion inhibits the development and progression of ductal neoplasia and prolongs survival in a murine model of pancreatic cancer. J Gastrointest Surg, 16, 104-12.

Donato R (2003). Intracellular and extracellular roles of S100 proteins. Microsc Res Tech, 60, 540-51.

Donato R, Cannon B, Sorci G, et al (2013). Functions of S100 proteins. Curr Mol Med, 13, 24-57.

Elangovan I, Thirugnanam S, Chen A, et al (2012). Targeting receptor for advanced glycation end products (RAGE) expression induces apoptosis and inhibits prostate tumor growth. Biochem Biophys Res Commun, 417, 1133-8.

Fritz G (2011). RAGE: a single receptor fits multiple ligands. Trends Biochem Sci, 36, 625-32.

Funk S, Mark R, Bayo P, et al (2014). High S100A8 and $\mathrm{S} 100 \mathrm{~A} 12$ protein expression is a favorable prognostic factor for survival of oropharyngeal squamous cell carcinoma. Int $J$ Cancer, [Epub ahead of print].

Gui Y, Guo G, Huang Y, et al (2011). Frequent mutations of chromatin remodeling genes in transitional cell carcinoma of the bladder. Nat Genet, $\mathbf{4 3}, 875-8$.

Gupta S, Hussain T, MacLennan GT, et al (2003). Differential expression of S100A2 and S100A4 during progression of human prostate adenocarcinoma. J Clin Oncol, 21, 106-12.

Heilmann RM, Wright ZM, Lanerie DJ, Suchodolski JS, Steiner JM (2014). Measurement of urinary canine S100A8/A9 and S100A12 concentrations as candidate biomarkers of lower urinary tract neoplasia in dogs. J Vet Diag Invest, 26, 104-12.

Kataoka K, Ono T, Murata H, et al (2012). S100A7 promotes the migration and invasion of osteosarcoma cells via the receptor for advanced glycation end products. Oncol Lett, 3, 1149-53.

Knowles MA, Hurst CD (2015). Molecular biology of bladder cancer: new insights into pathogenesis and clinical diversity. Nat Rev Cancer, 15, 25-41.

Lee S-J, Lee E-J, Kim S-K, et al (2012). Identification of proinflammatory cytokines associated with muscle invasive bladder cancer; the roles of IL-5, IL-20, and IL-28A. PLoS ONE, 7, 40267 .

Luo A, Kong J, Hu G, et al (2004). Discovery of Ca2 \pm relevant and differentiation-associated genes downregulated in esophageal squamous cell carcinoma using cDNA microarray. Oncogene, 23, 1291-9.

Matsumoto K, Irie A, Satoh T, et al (2007). Expression of S100A2 and S100A4 predicts for disease progression and patient survival in bladder cancer. Urology, 70, 602-7.

McKiernan E, McDermott EW, Evoy D, Crown J, Duffy MJ (2011). The role of S100 genes in breast cancer progression. Tumor Biol, 32, 441-50.

Meijer B, Gearry R, Day A (2012). The role of S100A12 as a systemic marker of inflammation. Int J Inflam, 2012, 907078.

Memon AA, Sorensen BS, Meldgaard P, et al (2005). Downregulation of $\mathrm{S} 100 \mathrm{C}$ is associated with bladder cancer progression and poor survival. Clin Cancer Res, 11, 606-11.

Nagy N, Brenner C, Markadieu N, et al (2001). S100A2, a putative tumor suppressor gene, regulates in vitro squamous cell carcinoma migration. Lab Invest, 81, 599-612.

Pietzsch J (2011). S100 proteins in health and disease. Amino Acids, 41, 755-60.

Ranasinghe WK, De Silva D, De Silva M, et al (2012). Incidence of bladder cancer in sri lanka: analysis of the cancer registry data and review of the incidence of bladder cancer in the South asian population. Korean J Urol, 53, 304-9.

Salama I, Malone PS, Mihaimeed F, Jones JL (2008). A review of the S100 proteins in cancer. Eur J Surg Oncol, 34, 357-64.

Salehi A, Khezri A, Malekmakan L, Aminsharifi A (2011). 
Epidemiologic status of bladder cancer in Shiraz, southern Iran. Asian Pac J Cancer Prev, 12, 1323-7.

Sessa L, Gatti E, Zeni F, et al (2014). The receptor for advanced glycation end-products (RAGE) is only present in mammals, and belongs to a family of cell adhesion molecules (CAMs). PLOS ONE, 9, 86903.

Shakhssalim N, Hosseini SY, Basiri A, et al (2010). Prominent bladder cancer risk factors in Iran. Asian Pac J Cancer Prev, 11, 601-6.

Xie J, Mendez JD, Mendez-Valenzuela V, Aguilar-Hernandez MM (2013). Cellular signalling of the receptor for advanced glycation end products (RAGE). Cell Signal, 25, 2185-97.

Yao R, Davidson D, Lopez Beltran A, et al (2007a). The S100 proteins for screening and prognostic grading of bladder cancer. Histol Histopathol, 22, 1025-32.

Yao R, Lopez-Beltran A, Maclennan GT, et al (2007b). Expression of $\mathrm{S} 100$ protein family members in the pathogenesis of bladder tumors. Anticancer Res, 27, 3051-8.

Yaser AM, Huang Y, Zhou RR, et al (2012). The role of receptor for advanced glycation end products (RAGE) in the proliferation of hepatocellular carcinoma. Int J Mol Sci, 13, 5982-97.

Yin C, Li H, Zhang B, et al (2013). RAGE-binding S100A8/ A9 promotes the migration and invasion of human breast cancer cells through actin polymerization and epithelialmesenchymal transition. Breast Cancer Res Treat, 142, 297-309.

Zhang G,Li M,Jin J,Bai Y, Yang C (2011). Knockdown of S100A4 decreases tumorigenesis and metastasis in osteosarcoma cells by repression of matrix metalloproteinase-9. Asian Pac $J$ Cancer Prev, 12, 2075-80.

Zhao CB, Bao JM, Lu YJ, et al (2014). Co-expression of RAGE and HMGB1 is associated with cancer progression and poor patient outcome of prostate cancer. Am J Cancer Res, 4, 369-77.

Zhao FT, Jia ZS, Yang Q, Song L, Jiang XJ (2013). S100A14 promotes the growth and metastasis of hepatocellular carcinoma. Asian Pac J Cancer Prev, 14, 3831-6. 